\title{
MINHO robot football team for 2001
}

\author{
Fernando Ribeiro, Carlos Machado, Sérgio Sampaio, Bruno Martins \\ Grupo de Automação e Robótica, Departamento de Electrónica Industrial, \\ Universidade do Minho, Campus de Azurém, 4800 Guimarães, Portugal \\ \{fernando.ribeiro, carlos.machado\}@dei.uminho.pt, \\ sergio.sampaio@mail.telepac.pt, brunomartins@bitmega.com
}

\begin{abstract}
This paper describes an autonomous robot football team. The work is being carried out since 1998. It describes the hardware used by the robots, the sensory system and interfaces, as well as the game strategy. Data acquisition for the perception level is carried out by the vision system, and the image processing system is described. Two cameras are used requiring sensorial fusion. With this architecture, an attempted is made to make the autonomous robots more real world intelligent. These robots have a kicker with controlled power, which allows passing the ball to a teammate with controlled distance and direction.
\end{abstract}

\section{Introduction}

This team already participated on previous RoboCup editions and has as main goal to develop a robotic team capable of playing football as comparable as possible to humans. Previous participation's allowed this team to learn and improve the robots and these are becoming more and more reliable. The most important improvements are: (a) new mechanical platform more reliable and robust; (b) kicker with controlled strength; (c) faster motor control with the use of dynamic algorithms; (d) self localization with one camera only; (e) use of two cameras per robot, requiring sensorial fusion but allowing an improved perception level; (f) improved color segmentation and acquisition. The next step is to improve the cooperation between the robots. For all these reasons, this team of robots is playing better than ever.

\section{Hardware Architecture}

A new robot was built from scratch, following the know-how acquired on previous competitions, and it was tested in a Portuguese championship confirming all the changes were successful. Now, the other three robots are being built. These new robots are below described.

The hardware is split in two separate parts: head and tail. The head contains all the power electronics, the electronic control boards, the computer and the vision system. The tail contains the chassis, the actuators (two motors and kicker), the motor gearboxes with encoders, the batteries and a device for ball control, hereby known as fork. 


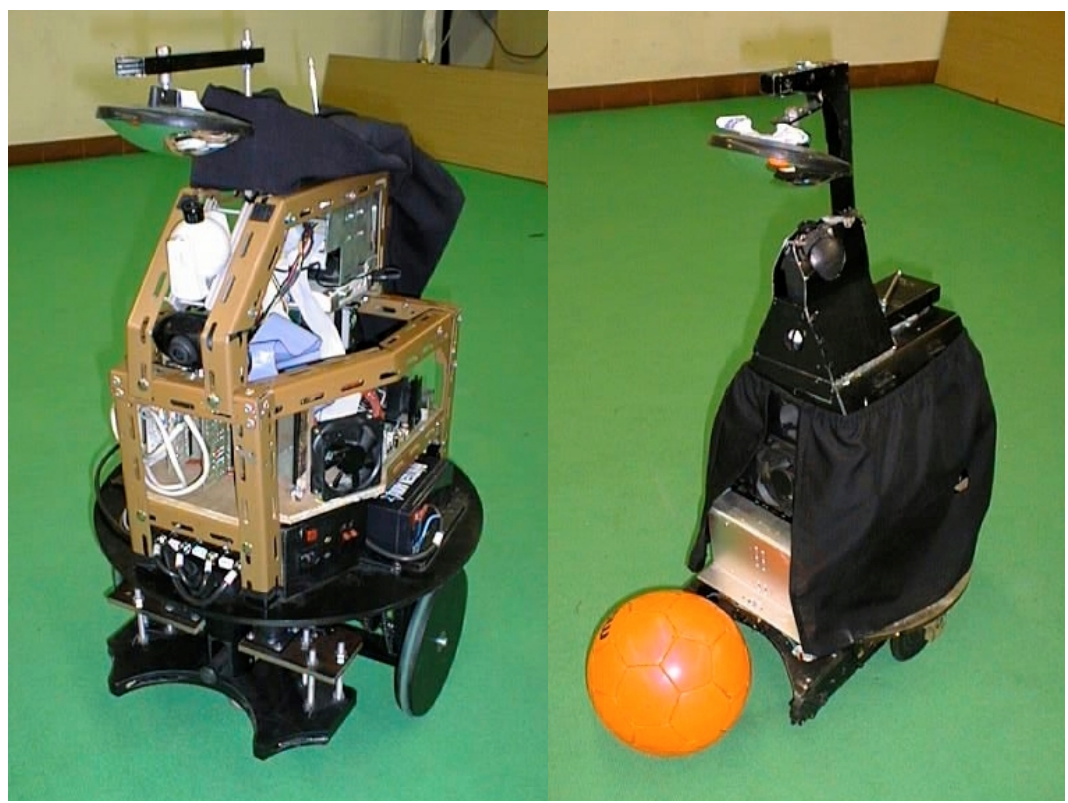

Fig. 1. Robots from MINHO team (new robot on the left, old robot on the right)

The old robots are still in use as testing platforms and are able to play football. Their description can be read in [1] and [2].

Table 1. Specifications of the new robot

\begin{tabular}{ll}
\hline Diameter & $50 \mathrm{~cm}$ \\
Height & $75 \mathrm{~cm}$ \\
Weight & $38 \mathrm{~kg}$. (incl.12Kg batteries) \\
Battery Power & 4 batteries of $12 \mathrm{~V} / 7 \mathrm{Ah}$ \\
drive & 2 wheel differential \\
Speed & $1.4 \mathrm{~m} / \mathrm{sec}$ \\
Motors & $6 \mathrm{~V} \mathrm{motors,} 8500 \mathrm{rpm}$ \\
Gearbox & ratio of $1 / 64$ \\
Encoder Resolution & 64 counts $/ \mathrm{ver}(9,8 \mathrm{~mm}$ per count $)$ \\
\hline
\end{tabular}

\subsection{The head}

The computer consists of a typical motherboard, with a Pentium II microprocessor running at $233 \mathrm{MHz}$, with 32 Mbytes of Memory, and a 4 Gbytes hard disk. The two serial and one parallel ports are used to communicate with the motors, kicker and encoders. Three motherboard slots are used; the VGA graphics card, the wireless network board from Lucent Technologies (using a $2.4 \mathrm{GHz}$ frequency) and a booktree bt848 chip based frame grabber where the two cameras are connected to (one outputs 
an S-video signal and the other Composite video). The top camera points to a convex parabolic shape mirror mounted over the camera and points downwards, and the front camera points forward. While the top camera gives an image from all around the robot, the front camera shows an image where game entities are larger and not distorted.

\subsection{The tail: Actuators}

The only actuators used by these robots are the motors and the kicker. This robot uses differential drive with two extra wheels to support the structure. The motors are capable of $8500 \mathrm{rpm}$ when fed continuously giving an output (after a gearbox reduction of 1/64) of $134 \mathrm{rpm}$ on the $200 \mathrm{~mm}$ diameter wheels, allowing speeds of up to 5 $\mathrm{Km} /$ hour. It is important to point out that the collision avoidance routines are extremely reliable and efficient.

The kicker consists of an electric wire coil, through which a current is passed, forming a magnetic field strong enough to move forward an iron core, which kicks the ball. The software controls the amount of time the current passes through, allowing to kick the ball to a desired distance. The formula to calculate the time of kick is:

$$
\text { time_of_kick }=(\text { desired_distance_in_metres }+5,5) / 0,225 \text {. }
$$

The shorter the distance, the slower the ball moves, the more unreliable the ball direction gets. But for distances above 1,5 meters the direction is perfectly acceptable.

These robots have a device to minimally control the ball hereby called fork, consisting of a round reentrance of only $7 \mathrm{~cm}$ (allowed by the rules). For safety and efficiency reasons, an infrared sensor is used between these two fork fingers which detect the presence of the ball when it obstructs the signal. The software contains a rule, which permits the kicker to be actuated ONLY if the RED ball is detected in the fork.

\subsection{The Tail: Sensors}

These robots use only two different physical sensors: an encoder coupled on each motor (to measure the number of turns of each motor) and an infrared transmitter and receptor, to detect the presence of the ball on the fork. In case the robot loses any of these sensors it is still able to play the game. All the information on the field needed to play a football game is percept by the vision system, rather than by any other physical sensors. The vision system apparatus was described above (Hardware: head), and therefore only the image processing software will now be described.

\section{Image Processing and game strategy}

Around about 20 frames per second are achieved on each camera, and the image is delivered on the YUV format, with a resolution of $384 \times 288$ pixels. Every pixel is converted into one of the 8 known colors with 32 levels, according to a Look up Table, built when calibrating the colors in a real football field. Then, the maximums of 
red and blue or yellow (depending on side attacking) are calculated and a threshold is applied, to avoid noise. All gray pixels are considered obstacles. On the image, virtual bumpers are used, which consist of 9 squares located around the robot and above a certain amount of gray inside, they consider an obstacle in that location.

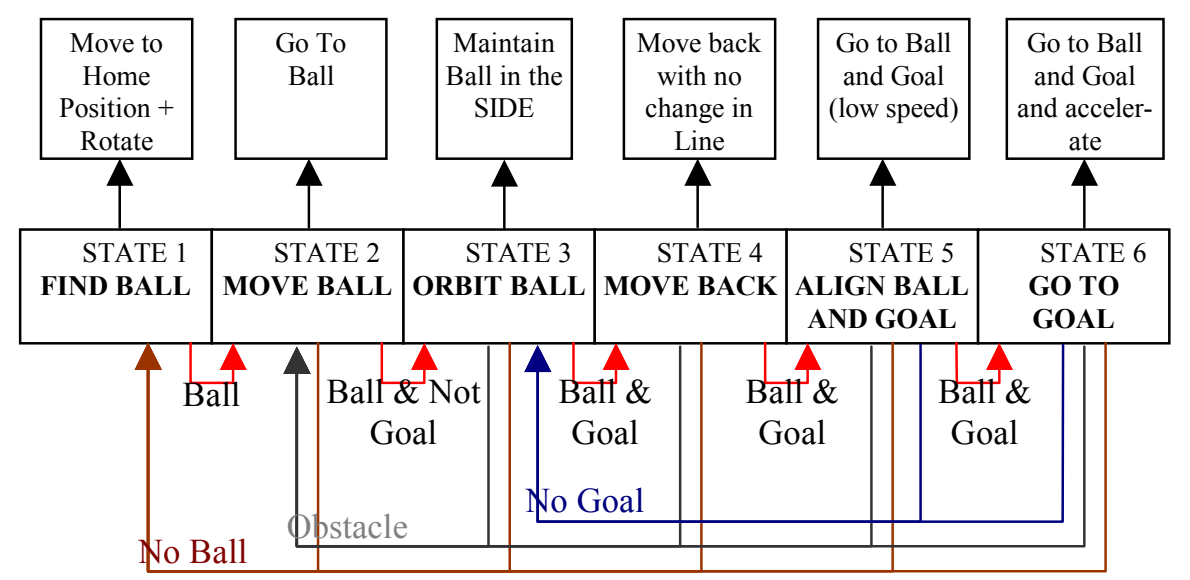

Fig. 2. Game Strategy

The game strategy is described in the figure above and consists of searching the ball, move towards the ball, once near the ball orbiting until the goal is in the field of view, move backwards to orient the robot, align the ball towards the goal, go towards the ball. Automatically, when the kicker "feels" the ball (with the infrared sensor) and the image "sees" the goal in front, it kicks the ball (known as instinct routine).

Self-localization is also achieved by analyzing the image of the front camera. A few transitions from green to white are seek on the image and according to the inclination of the line which passes by the transition coordinates, the location is calculated.

\section{Conclusion}

This team main areas of research are image processing and color segmentation, cooperative autonomous behaviors in multi-robot systems, self localization and distributed sensor fusion. Several improvements have been made in these new robots what concerns electronics, control, color segmentation and software strategy. These robots have been tested in some real games and proved to be mechanically reliable, with consistent play and scored many goals.

\section{References}

1. Sérgio Sampaio, Carlos Machado, Ilídio Costa, António Ribeiro, 'Patriarcas/Minho football team', RoboCup_99 Robot World Cup Soccer Games and Conference IJCAI'99, Teams Description Small and Middle Leagues, pag. 175-180, ISSN: 1401-9841, July 1999, Sweden. 